Gut and Liver, Vol. 9, No. 5, September 2015, pp. 569-570

\title{
Endoscopic Retrograde Cholangiopancreatography during Pregnancy: Really Guarantee to Safety?
}

\author{
Eun Taek Park \\ Division of Gastroenterology, Department of Internal Medicine, Kosin University School of Medicine, Busan, Korea
}

See "Efficacy and Safety of Pancreatobiliary Endoscopic Procedures during Pregnancy" by Jae Joon Lee, et al. on page 672 , Vol. 9. No. 5, 2015

Acute cholangitis and biliary pancreatitis caused by bile duct stones are not infrequently develop during pregnancy, they can cause serious complications and wastage for both mother and fetus. ${ }^{1}$ For pancreaticobiliary diseases in pregnancy, endoscopic retrograde cholangiopancreatography (ERCP) has been suggested as an effective alternative to surgery. ${ }^{2}$

During the procedure, radiation exposure is essentially develop on patient and subsequently exposed to the fetus can result in unpredictable future. These can bring a vague anxiety for patients and operators with regard to the procedure. ${ }^{3}$

In this issue of Gut and Liver, Lee et al. ${ }^{4}$ present that ERCP during pregnancy is relatively safe based on check the abnormality of the fetus in the relatively long-term follow-up period after the procedure and major complications associated with the procedure has not occurred. In addition, authors study shows that the ancillary diagnosis using endoscopic ultrasound (EUS) make a more safe and secure to help ERCP procedure when the patient complaint the symptoms obviously with difficult to diagnose the definitive cause. Also the study shows that just applying the shortening the procedure time, pregnant women to wear protectors and small amount of radiation exposure using the method of reducing the radiation exposure of the fetus, whereby they are possible to reach below the level of the international standards for exposure cause a serious problem. According to recently published papers related to the ERCP in the event that the radiation exposure of the fetus, the radiation dose to the fetus exposed is decreased through detailed and attentive care. $^{5,6}$ That is, limit X-ray beam on-time, limit the number of recorded images acquired and adjust the patient position (supine, prone, or lateral) to minimize fetal exposure.
According to published articles, ${ }^{7-9}$ some authors reported removed common bile duct stones with two-step ERCP for pregnant women according to the stage of pregnancy. If the patients were in late pregnancy, the stones were removed through a second ERCP after the pregnancy was terminated. If the patients were in early or mid-pregnancy, they underwent endoscopic retrograde biliary drainage and continued gestation. Their stents and stones were removed through a third ERCP 1 week after parturition, whereas others reported sphincterotomy with removing bile duct stone during the first ERCP procedure. The two groups were all safe procedures on maternal and fetal status and they did not show statistically significant complication rate related to procedure compare to nonpregnant group. In particular, sphincterotomy itself is secure whether or not the drain tube can be inserted and that significant results are reported to help preventing recurrent cholangitis and biliary pancreatitis.

In case of the patients are constantly complaining of biliary pain after performing ERCP, if the cause is confident to face the gallbladder or acute cholecystitis, undergo preventive cholecystectomy is reasonable. In contrast, the gallbladder stones without symptoms, first trimester and bile duct obstruction with stricture itself are recommended to delay surgery. In other words, we should apply in more stringent indications, rather than just indications of cholecystectomy applied in the nonpregnant group. ${ }^{10}$

If you plan to diagnostic ERCP with treatment due to doubt situation, it will be needed to longstanding procedure time, post-ERCP severe acute pancreatitis and eventually develop maternal and fetal risk. We should use nonradiation exposure techniques for the diagnosis of biliary stones such as EUS, magnetic

Correspondence to: Eun Taek Park

Division of Gastroenterology, Department of Internal Medicine, Kosin University Gospel Hospital, Kosin University School of Medicine, 262 Gamcheon-ro, Seo-gu, Busan 602-702, Korea

Tel: +82-51-990-6214, Fax: +82-51-990-5055, E-mail: euntpark@hanmail.net

pISSN 1976-2283 eISSN 2005-1212 http://dx.doi.org/10.5009/gnl15292

@ This is an Open Access article distributed under the terms of the Creative Commons Attribution Non-Commercial License (http://creativecommons.org/licenses/by-nc/4.0) which permits unrestricted non-commercial use, distribution, and reproduction in any medium, provided the original work is properly cited. 
resonance cholangiopancreatography, and EUS appropriately, thereby it is very important to know whether the ERCP indications are secure. ${ }^{11}$

In conclusion, ERCP for pancreatobiliary disease during pregnancy can be safe and effective procedure regardless of the period of pregnancy. The using guidelines related to radiation exposure protection in this study are to meet current several international standard recommendations.

\section{CONFLICTS OF INTEREST}

No potential conflict of interest relevant to this article was reported.

\section{REFERENCES}

1. Smith I, Gaidhane M, Goode A, Kahaleh M. Safety of endoscopic retrograde cholangiopancreatography in pregnancy: fluoroscopy time and fetal exposure, does it matter? World J Gastrointest Endosc 2013;5:148-153.

2. Cappell MS. The fetal safety and clinical efficacy of gastrointestinal endoscopy during pregnancy. Gastroenterol Clin North Am 2003;32:123-179.

3. Brent RL. The effect of embryonic and fetal exposure to x-ray, microwaves, and ultrasound: counseling the pregnant and nonpregnant patient about these risks. Semin Oncol 1989;16:347-368.
4. Lee JJ, Lee SK, Kim SH, et al. Efficacy and safety of pancreatobiliary endoscopic procedures during pregnancy. Gut Liver 2015;9: 672-678.

5. Samara ET, Stratakis J, Enele Melono JM, Mouzas IA, Perisinakis $\mathrm{K}$, Damilakis J. Therapeutic ERCP and pregnancy: is the radiation risk for the conceptus trivial? Gastrointest Endosc 2009;69:824831.

6. Baron TH, Schueler BA. Pregnancy and radiation exposure during therapeutic ERCP: time to put the baby to bed? Gastrointest Endosc 2009;69:832-834.

7. Tham TC, Vandervoort J, Wong RC, et al. Safety of ERCP during pregnancy. Am J Gastroenterol 2003;98:308-311.

8. Tang SJ, Mayo MJ, Rodriguez-Frias E, et al. Safety and utility of ERCP during pregnancy. Gastrointest Endosc 2009;69(3 Pt 1):453461

9. Yang J, Zhang X, Zhang X. Therapeutic efficacy of endoscopic retrograde cholangiopancreatography among pregnant women with severe acute biliary pancreatitis. J Laparoendosc Adv Surg Tech A 2013;23:437-440.

10. Patel SG, Veverka TJ. Laparoscopic cholecystectomy in pregnancy. Curr Surg 2002;59:74-78.

11. Lee YT, Chan FK, Leung WK, et al. Comparison of EUS and ERCP in the investigation with suspected biliary obstruction caused by choledocholithiasis: a randomized study. Gastrointest Endosc 2008;67:660-668. 